\title{
ON THE YIELD OF AN INTEREST RATE MODEL CALIBRATED WITH CURRENT TERM STRUCTURE
}

\author{
Man M. Chawla \\ X-027, Regency Park II, DLF City Phase IV \\ Gurgaon-122002, Haryana, INDIA
}

\begin{abstract}
It is desired of an interest rate model to be consistent with spot rates in the market. On the other hand, of interest is the behavior of asymptotic yield when the model is calibrated with initial term structure. In the present paper we show the interesting result that for an interest rate model calibrated with initial term structure, asymptotic yield of the resulting model is identical with initial forward rate, independent of the parameters involved in its short rate model. The result is first established for the Vasicek model using exact analysis and then, for a general four-parameter model where exact analysis seems intractable, through asymptotic analysis.
\end{abstract}

AMS Subject Classification: 91B24, 91B28, 91B30

Key Words: interest rate models, calibration with initial term structure, asymptotic yield, initial forward rate, Vasicek model, general four-parameter model

\section{Introduction}

A wide class of interest rate models is based on the dynamics of the short rate. Vasicek [6] is a classic interest rate model based on the three-parameter short rate model:

$$
d r=a(b-r) d t+\sigma d X .
$$

Since Vasicek model can give negative interest rates, a more popular model obviating this deficiency of the Vasicek model, is the Cox-Ingersoll-Ross [3]

Received: April 4, 2016

Published: June 20, 2016 (c) 2016 Academic Publications, Ltd.

url: www.acadpubl.eu 
interest rate model based on the short rate model:

$$
d r=a(b-r) d t+\sigma \sqrt{r} d X .
$$

Since then a very general four-parameter interest rate model has been proposed and studied based on the short rate model:

$$
d r=u(r, t) d t+w(r, t) d X
$$

where

$$
w(r, t)=\sqrt{\alpha r-\beta}, \quad u(r, t)=(\eta-\gamma r)+\lambda w(r, t) .
$$

See Klugman [4] and Klugman and Wilmott [5]. For a text-book discussion, see Wilmott et al [7]. Complete solutions for this four-parameter interest model are given in Chawla [1] and Chawla [2].

It is desired of an interest rate model to be consistent with spot rates in the market. On the other hand, of interest is the behavior of asymptotic yield of an interest rate model when the model is calibrated with initial term structure. While classical interest models of Vasicek and Cox-Ingersoll-Ross do not possess this property, a number of variations of the Vasicek type models have been proposed for the purpose.

In the present paper we show the interesting result that for an interest rate model calibrated with initial term structure, asymptotic yield of the resulting model is identical with the initial forward rate, independent of what and how many parameters are involved in its short rate model. This result is first established for the Vasicek model using exact analysis and then, for the general four-parameter model where exact analysis seems intractable, through asymptotic analysis.

For reference in the following sections, we note here some results for the four-parameter interest rate model.

For the four-parameter short rate model (1.3), the zero-coupon bond pricing equation giving the value $B(t, T)$ of a bond with maturity value one at time $T$ is

$$
B_{t}+\frac{1}{2}(\alpha r-\beta) B_{r r}+(\eta-\gamma r) B_{r}-r B=0 .
$$

For affine-yield solutions of the bond pricing equations:

$$
B(t, T)=e^{A(t, T)-r C(t, T)},
$$

differential equations for the determination of $A$ and $C$ are

$$
\frac{\partial A}{\partial t}=\eta C+\frac{1}{2} \beta C^{2}
$$




$$
\frac{\partial C}{\partial t}=\frac{1}{2} \alpha C^{2}+\gamma C-1
$$

with final conditions $A(T, T)=0, C(T, T)=0$. Once $C$ is found from (1.7), the corresponding $A$ is given, by integrating (1.6) from $t \rightarrow T$, by

$$
A(t, T)=-\eta \int_{t}^{T} C(s, T) d s-\frac{1}{2} \beta \int_{t}^{T} C^{2}(s, T) d s,
$$

for constant $\eta$. With time to maturity $\tau=T-t$, the yield is

$$
Y(t, T)=-\frac{1}{\tau} \ln B(t, T),
$$

The function $Y(t, T)$ of two variables, $t<T$, is term structure of interest rates while yields $Y(0, T)$ dictated by current prices are the spot rates. Now, the initial forward rate at time $\mathrm{t}=0$, in terms of initial term structure or yields, is given by

$$
f(0, t, T)=\frac{1}{\tau}\left[T Y_{0}(T)-t Y_{0}(t)\right]
$$

Note that in terms of the affine-yield solution yield (1.9) can be written as

$$
Y(t, T)=\frac{1}{\tau}[-A(t, T)+r C(t, T)] .
$$

We finally note that the Vasicek and Cox-Ingersoll-Ross models are included as particular cases in the four-parameter interest rate model respectively for $\alpha=0$ and $\beta=0$.

\section{Proof for the Vasicek Model}

For the Vasicek model, $C$ is given by

$$
C(\tau)=\frac{1-e^{-\gamma \tau}}{\gamma}
$$

and the corresponding $A$ is given by

$$
A(t, T)=-\eta I(t, T)-\frac{1}{2} \beta J(t, T),
$$

where

$$
I(t, T)=\int_{t}^{T} C(s, T) d s, J(t, T)=\int_{t}^{T} C^{2}(s, T) d s .
$$


Since

$$
I(t, T)=\frac{1}{\gamma}(\tau-C(\tau)), J(t, T)=\frac{1}{\gamma^{2}}\left(\tau-C(\tau)-\frac{\gamma}{2} C^{2}(\tau)\right),
$$

therefore,

$$
A(t, T)=\frac{1}{\gamma}\left(\eta+\frac{\beta}{2 \gamma}\right)[C(\tau)-\tau]+\frac{\beta}{4 \gamma} C^{2}(\tau) .
$$

The yield curve is given by

$$
Y(t, T)=\frac{1}{\gamma}\left(\eta+\frac{\beta}{2 \gamma}\right)\left[1-\frac{C(\tau)}{\tau}\right]-\frac{\beta}{4 \gamma} \frac{C^{2}(\tau)}{\tau}+\frac{r}{\tau} C(\tau),
$$

with asymptotic, for $\tau \rightarrow \infty$, behavior

$$
Y(t, T) \sim \frac{1}{\gamma}\left(\eta+\frac{\beta}{2 \gamma}\right) .
$$

To fit a yield curve in the Vasicek model, we treat $\eta$ as a function of time and write (1.8) as

$$
A(t, T)=-\int_{t}^{T} \eta(s) C(s, T) d s-\frac{1}{2} \beta J(t, T) .
$$

To fit the initial yield curve $Y_{0}(T)$ for time $t=0$, we initialize (2.5) for time $t=0$ :

$$
A_{0}(T)=-\int_{0}^{T} \eta(s) C(s, T) d s-\frac{1}{2} \beta J_{0}(T),
$$

substitute for $A_{0}(T)$ in terms of the initial yield curve $Y_{0}(T)$ from (1.10) and obtain

$$
\int_{0}^{T} \eta(s)\left(1-e^{-\gamma(T-s)}\right) d s=\gamma F_{0}(T),
$$

where we have set

$$
F_{0}(T)=T Y_{0}(T)-r C_{0}(T)-\frac{1}{2} \beta J_{0}((T) .
$$

Differentiating (2.6) with respect to $T$, using the differentiation rule:

$$
\frac{d}{d x} \int_{a}^{x} f(s, x) d s=f(x, x)+\int_{a}^{x} \frac{d}{d x} f(s, x) d s,
$$

and adding the resulting integral to (2.6) we get

$$
\int_{0}^{T} \eta(s) d s=\gamma F_{0}(T)+F_{0}^{\prime}(T) .
$$


One more differentiation with respect to $T$ gives the required

$$
\eta(T)=\gamma F_{0}^{\prime}(T)+F_{0}^{\prime \prime}(T)
$$

To find the corresponding $A^{*}(t, T)$ from $(2.5)$, we first evaluate

$$
K(t, T)=\int_{t}^{T}\left[\gamma F_{0}^{\prime}(s)+F_{0}^{\prime \prime}(s)\right] C(s, T) d s
$$

Integrating the second term by parts and simplifying we get

$$
K(t, T)=F_{0}(T)-F_{0}(t)-F_{0}^{\prime}(t) C(\tau)
$$

Since

$$
\begin{gathered}
C_{0}(T)-C_{0}(t)=e^{-\gamma t} C(\tau), \\
J(t, T)-\left(J_{0}(T)-J_{0}(t)\right)=-C_{0}^{2}(t) C(\tau)-\frac{\gamma}{2}\left(1-e^{-2 \gamma t}\right) C^{2}(\tau),
\end{gathered}
$$

therefore with (2.7) and (2.9) from (2.5) we obtain

$$
\begin{aligned}
A^{*}(t, T)= & -\tau f(0, t, T)+r e^{-\gamma t} C(\tau)+F_{0}^{\prime}(t) C(\tau) \\
& +\frac{\beta}{2}\left[C_{0}^{2}(t) C(\tau)+\frac{\gamma}{2}\left(1-e^{-2 \gamma t}\right) C^{2}(\tau)\right] .
\end{aligned}
$$

The corresponding yield curve is

$$
Y^{*}(t, T)=\frac{1}{\tau}\left[-A^{*}(t, T)+r C(t, T)\right] .
$$

For asymptotic behavior of the yield, while all other terms go to zero, the only term containing $\tau$ contributing to asymptotic yield gives

$$
Y^{*}(t, T) \sim f(0, t, T)
$$

thus proving the result that for the Vasicek model calibrated with term structure, asymptotic yield is identical with the initial forward rate independent of what and how many parameters are involved in its short rate model (1.1). 


\section{Asymptotic Proof for the Vasicek Model}

With a view to validate asymptotic analysis of the next section, we consider here an asymptotic analysis to prove the result for the Vasicek model obtained in Section 2.

For $\tau \rightarrow \infty, C(t, T)$ for Vasicek model is given by

$$
C_{\infty}=\frac{1}{\gamma}
$$

with corresponding $A(t, T)$ given by

$$
A_{\infty}=-\frac{1}{\gamma}\left(\eta+\frac{\beta}{2 \gamma}\right) \tau .
$$

The yield curve is

$$
Y_{\infty}=\frac{1}{\tau}\left[-A_{\infty}+r C_{\infty}\right]=\frac{1}{\gamma}\left(\eta+\frac{\beta}{2 \gamma}\right),
$$

which agrees with the asymptotic yield for the Vasicek model in (2.4).

Next, to calibrate Vasicek model with the initial yield, we start with asymptotic form of (2.5):

$$
A_{\infty}(t, T)=-\frac{1}{\gamma} \int_{t}^{T} \eta(s) d s-\frac{1}{2} \beta J_{\infty}(t, T),
$$

where

$$
J_{\infty}(t, T)=\frac{1}{\gamma^{2}}\left(\tau-\frac{3}{2 \gamma}\right) .
$$

To fit initial yield curve at $t=0$, we initialize (3.1):

$$
A_{0, \infty}(T)=-\frac{1}{\gamma} \int_{0}^{T} \eta(s) d s-\frac{1}{2} \beta J_{0, \infty}(T) .
$$

Substituting for $A_{0, \infty}$ in terms of initial yield curve from (1.9) we have

$$
\int_{0}^{T} \eta(s) d s=\gamma F_{0}(T),
$$

where we have set

$$
F_{0}(T)=T Y_{0}(T)-\frac{r}{\gamma}-\frac{1}{2} \beta J_{0, \infty}(T) .
$$


Differentiating with respect to $T$ gives

$$
\eta(T)=\gamma F_{0}^{\prime}(T)
$$

With this we have the corresponding $A_{\infty}^{*}$ from (3.1) as

$$
A_{\infty}^{*}(t, T)=-\left[F_{0}(T)-F_{0}(t)\right]-\frac{1}{2} \beta J_{\infty}(t, T) .
$$

Since

$$
F_{0}(T)-F_{0}(t)=f(0, t, T) \tau-\frac{\beta}{2 \gamma^{2}} \tau
$$

therefore,

$$
A_{\infty}^{*}(t, T)=-f(0, t, T) \tau+\frac{\beta}{2 \gamma^{2}} \tau-\frac{\beta}{2 \gamma^{2}}\left(\tau-\frac{3}{2 \gamma}\right)
$$

The yield curve is given by

$$
Y_{\infty}^{*}(t, T)=\frac{1}{\tau}\left[-A_{\infty}^{*}(t, T)+\frac{r}{\gamma}\right]
$$

Asymptotic behavior of the yield is

$$
Y_{\infty}^{*}(t, T) \sim f(0, t, T)-\frac{\beta}{2 \gamma^{2}}+\frac{\beta}{2 \gamma^{2}}=f(0, t, T),
$$

thus proving the result by this asymptotic analysis that in Vasicek model calibrated with initial term structure, the asymptotic yield is identical with initial forward rate independent of what and how many parameters are involved in its short rate model.

\section{Asymptotic Proof for the Four-Parameter Interest Rate Model}

As noted before, exact analysis of calibration of the four-parameter interest rate model with initial term structure seems intractable, so here we offer an asymptotic proof of the result that asymptotic yield of the calibrated model is identical with the initial forward rate. For the four-parameter interest rate model, the following notation has been used in Chawla [1] and Chawla [2]:

$$
\psi=\sqrt{\gamma^{2}+2 \alpha}, a=\frac{-\gamma+\psi}{\alpha}, b=\frac{\gamma+\psi}{\alpha} .
$$


From Chawla [2], for $\tau \rightarrow \infty$, asymptotic $C(t, T)$ is given by

$$
C_{\infty}=\frac{2}{\alpha b}=a
$$

with corresponding $A(t, T)$ given by

$$
A_{\infty}=-a\left(\eta+\frac{\beta a}{2}\right) \tau
$$

The yield curve is

$$
Y_{\infty}=\frac{1}{\tau}\left[-A_{\infty}+r C_{\infty}\right]=a\left(\eta+\frac{\beta a}{2}\right),
$$

which agrees with the asymptotic yield for the four-parameter interest rate model in Chawla [2].

Next, to calibrate the four-parameter model with the initial term structure, we start with asymptotic form of (2.5):

$$
A_{\infty}(t, T)=-a \int_{t}^{T} \eta(s) d s-\frac{\beta a^{2}}{2} \tau .
$$

To fit the initial yield curve for $t=0$, we initialize (4.1):

$$
A_{0, \infty}(T)=-a \int_{0}^{T} \eta(s) d s-\frac{\beta a^{2}}{2} T .
$$

Substituting for $A_{0, \infty}$ in terms of initial yield curve from (1.9) we have

$$
\int_{0}^{T} \eta(s) d s=\frac{1}{a} F_{0}(T),
$$

where we have set

$$
F_{0}(T)=T Y_{0}(T)-r a-\frac{\beta a^{2}}{2} T .
$$

Differentiating (4.2) with respect to $T$ gives

$$
\eta(T)=\frac{1}{a} F_{0}^{\prime}(T)
$$

With this we have the corresponding $A_{\infty}^{*}$ from (4.1) as

$$
A_{\infty}^{*}(t, T)=-\left[F_{0}(T)-F_{0}(t)\right]-\frac{\beta a^{2}}{2} \tau .
$$


Since

$$
F_{0}(T)-F_{0}(t)=f(0, t, T) \tau-\frac{\beta a^{2}}{2} \tau
$$

therefore,

$$
A_{\infty}^{*}(t, T)=-f(0, t, T) \tau .
$$

The yield curve is given by

$$
Y_{\infty}^{*}(t, T)=\frac{1}{\tau}\left[-A_{\infty}^{*}(t, T)+r a\right] .
$$

With (4.3), asymptotic value of the yield is

$$
Y_{\infty}^{*}(t, T) \sim f(0, t, T)
$$

thus proving the result by this asymptotic analysis that for the four-parameter interest rate model calibrated with initial term structure, the asymptotic yield is identical with the initial forward rate independent of what and how many parameters are involved in the its short rate model (1.3).

\section{References}

[1] M.M. Chawla, On solutions of the bond pricing equation, Int. J. Appl. Math., 23 (2010), 661-680.

[2] M.M. Chawla, On the four-parameter bond pricing model, Int. J. Appl. Math., 29 (2016), 53-68, doi: 10.12732/ijam.v29i1.5.

[3] J.C. Cox, J.E. Ingersoll, S.A. Ross, A theory of the term structure of interest rates, Econometrica, 53 (1985), 385-407.

[4] R. Klugman, Interest rate modelling, OCIAM working paper, Mathematical Institute, Oxford University, 1992.

[5] R. Klugman, P. Wilmott, A four parameter model for interest rates, OCIAM working paper, Mathematical Institute, Oxford University, 1993.

[6] O. Vasicek, An equilibrium characterization of the term structure, Journal of Financial Economics, 5 (1977), 177-188.

[7] P. Wilmott, S. Howison, J. Dewynne, The Mathematics of Financial Derivatives: A Student Introduction, Cambridge University Press, Cambridge, 1995. 
